\title{
Advanced Digital Beamforming Concepts for Future SAR Systems
}

G. Krieger, M. Younis, N. Gebert, S. Huber, F. Bordoni, A. Patyuchenko, A. Moreira

Microwaves and Radar Institute, German Aerospace Center (DLR), email: gerhard.krieger@dlr.de

\begin{abstract}
This paper reviews advanced multi-channel SAR system concepts for the imaging of wide swaths with high resolution. Several novel system architectures employing both direct radiating arrays and reflector antennas fed by a digital array are introduced and compared to each other with regard to their imaging performance. In addition, innovative SAR imaging modes are proposed which enable the mapping of ultra-wide swaths with high azimuth resolution. The new techniques and technologies have the potential to enhance the imaging performance of future SAR systems by one order of magnitude if compared to state of the art SAR sensors like TerraSAR-X, ALOS, Radarsat-2 or Sentinel-1.
\end{abstract}

\section{Introduction}

Wide unambiguous swath coverage and high azimuth resolution pose contradicting requirements on the design of spaceborne synthetic aperture radar (SAR) systems [1]. This motivated the development of advanced SAR imaging modes with different trade-offs between spatial coverage and azimuth resolution. Examples are the ScanSAR (or TOPS) mode which enables a wide swath at the cost of an impaired azimuth resolution and the Spotlight mode which allows for an improved azimuth resolution at the cost of a noncontiguous imaging along the satellite track. It is, however, up to now not possible to combine both operational modes simultaneously in one and the same data take. This dilemma motivated further research towards the development of new radar techniques for spaceborne high-resolution wide-swath SAR imaging.

\section{System Architectures}

Several proposals resolve the azimuth resolution vs. wide swath coverage dilemma by combining a multi-channel radar receiver with a small aperture transmitter illuminating a wide area on the ground. Examples are the squinted multiple beam SAR (Figure 2, left, [2]), the displaced phase centre antenna (DPCA) technique (Figure 2, second column, [3]), the Quad Array SAR system (Figure 2, third column, [4]), and the High-Resolution Wide-Swath (HRWS) SAR system (Figure 2, fourth column, [5]).

An interesting alternative to a direct radiating array is the combination of a reflector with a digital feed array as suggested in [6][7][8]. This architecture combines the capabilities of digital beamforming with the high gain provided by a large reflector aperture. A reflector antenna offers moreover the potential to use one and the same aperture for multiple frequency bands simultaneously, thereby paving the way for future multi-frequency SAR systems. To lower launch volume and weight, the reflector could be deployable as already suggested for several L-band radar missions [9][10][11]. Unfurlable reflectors are now a mature technology with extensive flight heritage in space telecommunications and satellites with lightweight mesh reflectors spanning diameters of more than $20 \mathrm{~m}$ will be launched in the near future [12].

The reflector based digital beamforming architecture offers several attractive features for the design of high performance spaceborne SAR systems. For example, the simultaneous activation of all feed elements generates a broad transmit beam for wide swath illumination. The red curve in the left plot of Figure 1 shows as an example the L-band transmit pattern of a $15 \mathrm{~m}$ reflector antenna with a $\mathrm{f} / \mathrm{d}$ of $2 / 3$ and a centre fed linear array consisting of 24 feed elements with a $0.6 \lambda$ spacing. The opportunity to use all feed elements simultaneously for the generation of a wide transmit beam avoids the necessity of a separate transmit antenna as suggested for the HRWS system and/or the use of sophisticated illumination strategies in case of using a combined $\mathrm{Tx} / \mathrm{Rx}$ array [13]. On the other hand, radar echoes arriving as plane waves from a given direction activate typically only one or a small number of feed elements if the feed array is located close to the focal plane.
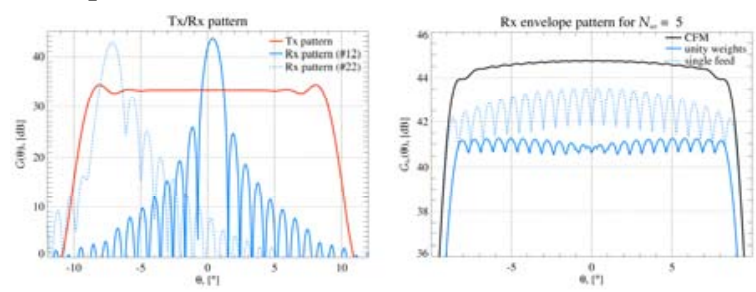

Figure 1 - Antenna patterns for a 15m L-band reflector with various feed excitations (cf. text)

The systematic correspondence between beam direction and activated feed array element(s) is well suited to significantly enhance the imaging performance of future side-looking radar sensors where the scattered radar echoes arrive at each instant of time only from a rather narrow angular range ${ }^{1}$. The angle of arrival migrates in synchrony with range time as the radar echoes arrive in successive order from near to far range. By using this correspondence, a significant improvement in the sensitivity (NESZ) and range ambiguity suppression can be achieved via a dynamic routing of those feed signal(s) that exceed a given threshold level. The solid and dotted blue curves in the centre plot of Figure 1

\footnotetext{
${ }^{1}$ For a long chirped transmit pulse, the radar echoes arrive from slightly different angles for each range frequency. Optimum sensitivity requires hence a "dispersive Rx beam". Such a beam can be synthesized by a bandpass decomposition (in range frequency) for each recorded feed element signal, followed by a different feed element combination for each sub-band. Simply speaking, this corresponds to a division of the echo from a long chirped Tx pulse into multiple echoes from short narrow-frequency pulses and an optimum beam can then be formed for each of these short sub-pulses.
} 


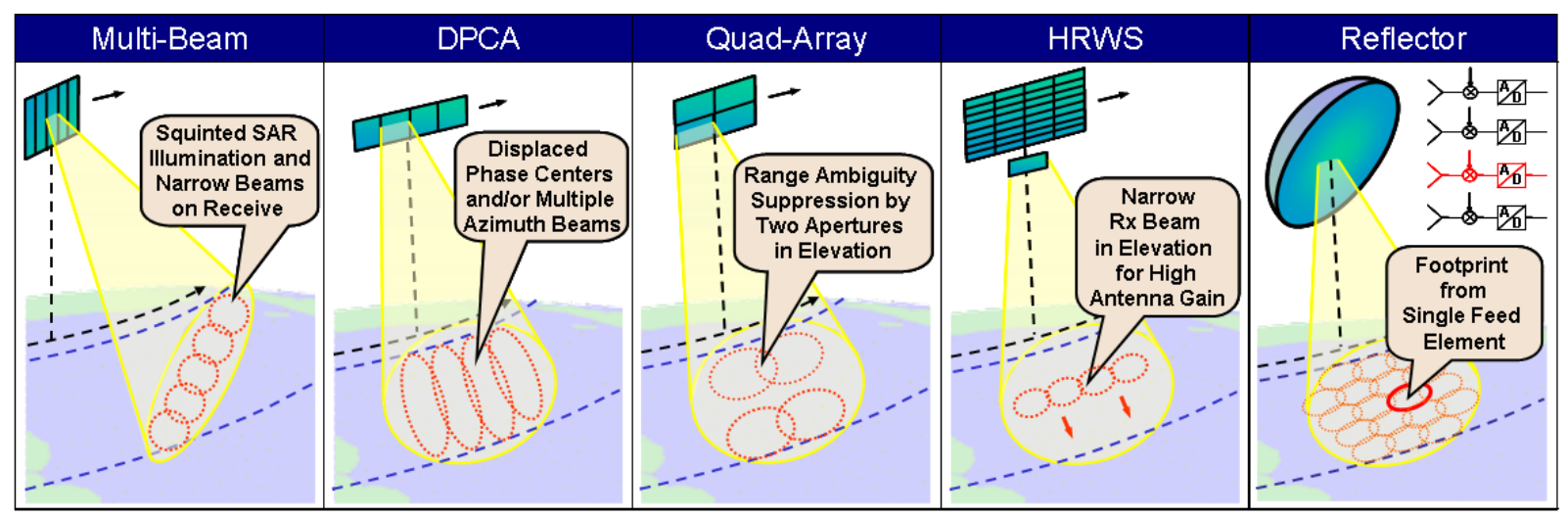

Figure 2- Solutions for high-resolution wide-swath SAR imaging. Left: multiple beam SAR. Middle left: displaced phase center antenna technique. Middle: quad-element rectangular array SAR. Middle right: highresolution wide-swath (HRWS) SAR. Right: High resolution wide swath SAR employing a reflector antenna in combination with a digital feed array.

show as an example the resulting antenna patterns for activating feed elements $\# 12$ and $\# 22$, respectively. It is clear that the gain of the narrow $\mathrm{Rx}$ pattern is about 10 $\mathrm{dB}$ higher than that of the broad Tx pattern, thereby compensating the inevitable gain loss from the illumination of a wide image swath.

A further improvement of the performance can be achieved by combining the signals from multiple feed elements. This can be seen on the right hand side of Figure 1 which shows the Rx "sensitivity" patterns for (1) selecting for each angle the element with maximum gain (dotted blue), (2) linear summation of the signals from 5 neighbouring elements with unity weights (solid blue), and (3) optimum phase and amplitude weighted combination of 5 neighbouring element signals (conjugate field matching, black solid line, see also [14]). It is evident that the digital combination of multiple feed element signals improves the performance and yields a higher and smoother Rx sensitivity pattern. The latter is desired to avoid phase jumps for interferometric applications and to relax calibration requirements.

Digital beamforming in a reflector system involves typically only the combination of a low number of array elements. This significantly reduces the on-board processing requirements if compared to a direct radiating array. For example, only 5 element signals have been combined at each instant of time in the conjugate field matching simulations of Figure 1. In contrast, a real time scanon-receive with a $15 \mathrm{~m}$ high planar array would require the onboard combination of more than 50 element signals. The increased performance achievable by a large reflector aperture relaxes also the satellite's thermal, power and energy demands and/or allows for longer operation times as desired for future Earth system monitoring missions which ask for long orbital duty cycles [15].

\section{Operational Modes}

The previous section discussed multi-channel architectures for future high-resolution wide-swath SAR systems. In the following, we introduce and compare different SAR imaging modes that take advantage of the new digital radar architectures. As a design example we consider a system which shall be able to map a swath width of approx. $400 \mathrm{~km}$ with an azimuth resolution of $5 \mathrm{~m}$. Such a system exceeds by far the capabilities of current spaceborne SAR sensors. To avoid a too strong variation of the incident angles we assume an orbital altitude of $750 \mathrm{~km}$.

\subsection{Multi-Channel Stripmap Mode}

We first consider a multi-aperture mapping in standard strip-map mode. A timing analysis reveals that the imaging of a contiguous $400 \mathrm{~km}$ swath requires a PRF of approximately $400 \mathrm{~Hz}$ [6]. To avoid azimuth ambiguities, the necessary antenna length can be approximated by $l_{\text {ant }} \approx 2 v /$ PRF which yields an antenna length of $l_{\text {ant }} \approx$ $35 \mathrm{~m}$. The azimuth resolution of a conventional single channel SAR would then be in the order of $\Delta a z \approx 20 \mathrm{~m}$. By illuminating a wider Doppler spectrum and dividing the receiver antenna into multiple azimuth apertures with individual receiver channels it becomes possible to improve the azimuth resolution to $\Delta a z \approx l_{\text {ant }} /\left(2 N_{\mathrm{az}}\right)$ where $N_{\mathrm{az}}$ is the number of independent azimuth channels. The desired azimuth resolution of $5 \mathrm{~m}$ would hence require at least $N_{\mathrm{az}}=4$ channels. Such a resolution improvement by multiple azimuth Rx channels is possible for both the direct radiating array and the reflector configuration. The performance gain can be understood in the former case as the acquisition of additional samples along the synthetic aperture and in the latter case as the acquisition of additional frequency sub-bands with different Doppler centroids to increase the overall azimuth bandwidth.

\subsection{Multi-Channel ScanSAR Mode}

The major drawback of the multi-channel stripmap mode is the long antenna required for unambiguous wide swath SAR imaging. An alternative is the ScanSAR or TOPS mode. Assuming an antenna length of $12 \mathrm{~m}$, the minimum PRF is in the order of $1.25 \mathrm{kHz}$. Timing considerations reveal that at least 4 bursts would be required to cover a $400 \mathrm{~km}$ swath. The azimuth resolution is then $\Delta a z>$ $\left(N_{\text {burst }}+1\right) \cdot l_{\text {ant }} / 2$ where $N_{\text {burst }}$ is the number of bursts which yields $\Delta a z>30 \mathrm{~m}$. To achieve an azimuth resolution of $\Delta a z=5 \mathrm{~m}$ one may again employ multiple azimuth chan- 
nels connected to displaced antenna elements. The minimum number of azimuth channels is $N_{a z}>N_{\text {burst }}+1$. Hence, at least 6 channels will be required, but typically this number is even higher due to the finite roll-of of the involved antenna patterns. Challenges arise for the multiaperture processing from the large squint angles, the varying target Doppler spectra, and the different burst PRFs. As a result, the azimuth ambiguity to signal ratio (AASR) shows a significant variation with both the azimuth position and the burst PRF. A detailed performance analysis together with an extension to a multi-channel TOPS mode can be found in [16].

\subsection{Multiple Beam SAR Modes}

The mapping of a wide image swath with a reasonable short antenna length requires, especially in fully polarimetric mode, a large number of bursts. This impairs the performance and leads to conflicts with regard to the achievable azimuth resolution. Such problems can be mitigated by a simultaneous mapping of multiple swaths during each burst as illustrated in Figure 3.

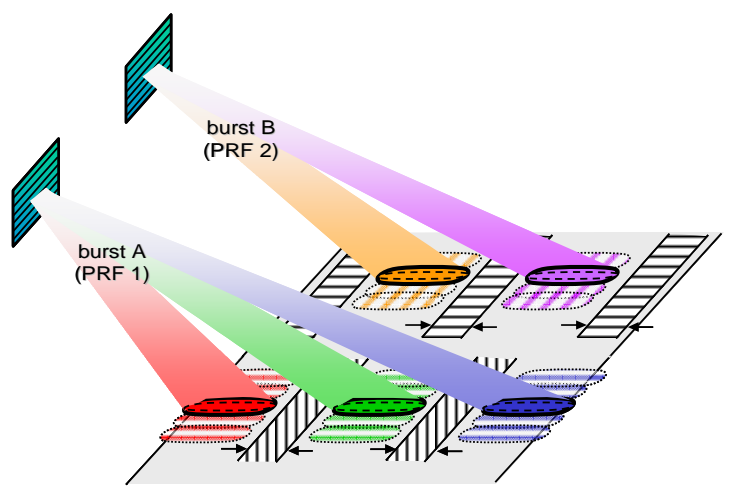

Figure 3 - SAR imaging with multiple beams in burst mode operation.

Two bursts with slightly different PRFs are sufficient to map an ultra-wide swath if range ambiguities and nadir echoes are suppressed by digital beamforming on receive in elevation. Here, a reflector based system could have the advantage to avoid possible saturation effects in the receiver channels that could otherwise arise for very strong nadir returns. The strength of the nadir signal can also be reduced by a careful design of the Tx antenna pattern and a further improvement is possible by using different waveforms for each transmitted pulse which enables a spread of the nadir energy in the received echoes.

The performance of the multiple beam ScanSAR mode can be improved by increasing the number of PRFs. The limit is a continuous variation of the PRF. As an example we consider a periodic linear increase of the PRF which shifts the blind ranges smoothly across the swath as illustrated in Figure 4. Note that a rather small variation of the PRF is already sufficient to obtain full wide swath coverage [6]. The red lines in Figure 4 indicate the duration of the bursts which is typically longer than in the classical ScanSAR mode. This improves the azimuth resolution. The SAR focusing of each burst requires an appropriate preprocessing which interpolates the (multi-channel) azimuth raw data to a uniform sampling interval.

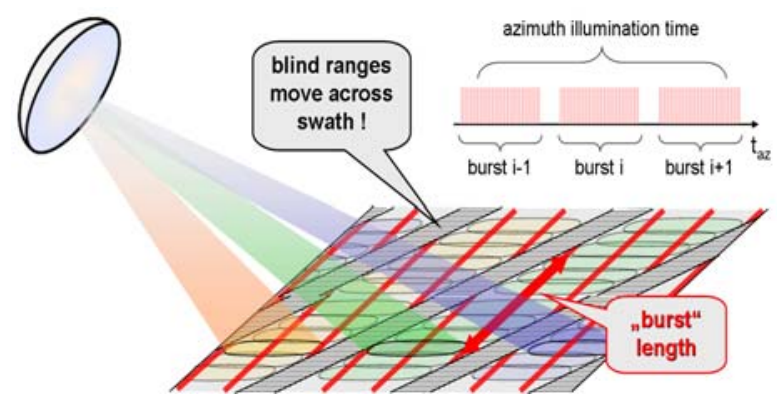

Figure 4 - Multiple swath imaging with variable PRF.

A further improvement arises in case of very short transmit pulses where one looses for each range only a rather short segment of the synthetic aperture. Hence, a coherent processing of the full azimuth spectrum can be considered. This yields a range independent azimuth resolution of approx. half the antenna length for an arbitrary swath width. The interpolation of the gaps will increase the integrated sidelobe ratio (ISLR) of the impulse response, but for short duty cycles this effect can be made sufficiently small [17].

The "multiple beam variable PRF" mode could be of special interest for a reflector based digital beamforming system where we can benefit from a high antenna for improved range ambiguity suppression. Moreover, a large reflector aperture allows also for a high Rx gain, thereby enabling the usage of very short Tx pulses as desired to minimize gap losses.

\subsection{Hybrid SAR Modes}

Often, the user requirements ask for high-resolution data takes within a small area while at the same time wide-swath coverage with coarser resolution is needed for frequent monitoring applications. The brute force approach to meet these requirements is the acquisition of very wide swaths with high geometric resolution as outlined in the previous sections. However, such a global-scale high-resolution wide-swath imaging is also associated with a huge data volume, thereby increasing the demands for internal data storage, downlink, ground processing and archiving. A promising solution to these challenges is the use of hybrid SAR modes [13][15]. These modes can be tailored to provide a variable resolution within the imaged scene (cf. Figure 5) and are hence suited to resolve user and application conflicts without an explosion of the data volume.

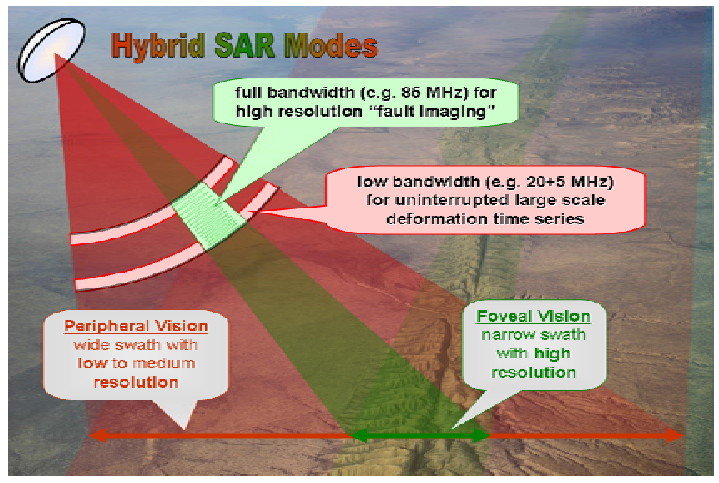

Figure 5 - Hybrid SAR modes enable radar imaging with variable resolution 
Hybrid modes can easily be implemented with a reflector based DBF architecture. The left plot in Figure 6 shows as an example the feed element activation pattern for a possible implementation of a hybrid SAR mode. In this example, a subset of the feed elements transmits a linear frequency modulated chirp signal of full bandwidth while the remaining feed elements transmit only a portion of the chirp but with the same chirp rate. The shortened pulse durations for most of the feed elements reduce the average transmit power if compared to a full high-resolution wide-swath SAR system.
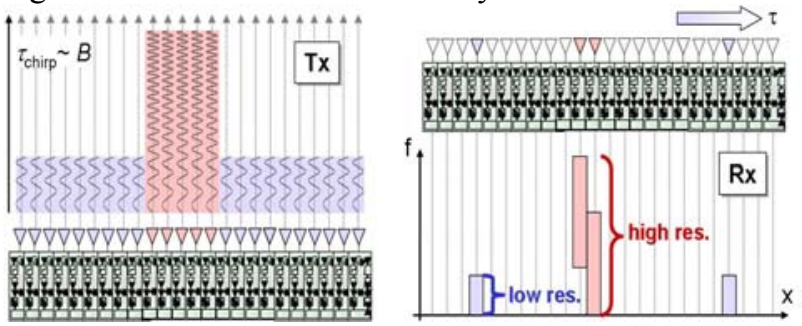

Figure 6 - (Left) Feed activation pattern for the transmission of a space-variant chirp bandwidth. (Right) Snapshot of the received signals in a space-frequency representation.

The recording of the scattered signals is shown in Figure 6 on the right. Only a small subset of the feed elements receives swath echoes at a given instant of time. From these feed elements, again only a small subset receives a full bandwidth signal while the residual activated feed elements receive a short narrow-band signal that can be sampled at a much lower frequency, thereby significantly reducing the overall data volume. The hybrid mapping of wide swaths requires in general also a systematic variation of the PRF to avoid blind ranges. One opportunity is the combination of a local highresolution stripmap acquisition with a wide-swath ScanSAR mode. Such a StripScan hybrid provides a non-homogeneous resolution in azimuth and allows for a further reduction of the data volume. For this, the feed elements illuminating the wide swath are operated in bursts. This reduces both the average Tx power and the number of simultaneously activated feeds during signal reception. The hybrid of stripmap and ScanSAR makes the PRF selection more stringent, since one has to avoid gaps in both the stripmap and the ScanSAR sub-swaths. Suitable PRFs can be found since the high resolution area covers only a portion of a full stripmap swath. The timing benefits moreover from the short Tx pulses and the active nadir echo suppression via the narrow $\mathrm{Rx}$ beams. As an alternative, one may again consider a continuous variation of the PRF as outlined in Section 3.3.

\section{Conclusions}

We have introduced several new SAR system concepts for the acquisition of high resolution radar images with ultra wide swath coverage. The innovative concepts rely on the combination of advanced multi-channel radar front-end architectures with novel operational modes. It becomes clear that from a signal theoretical point of view it is possible to design high-resolution ultra-wide-swath SAR sensors with rather compact antennas.
Regarding implementation complexity, it was shown that deployable reflector antennas are an interesting alternative to direct radiating arrays and a low number of receiver channels are already sufficient to enhance the imaging capabilities far beyond those of current spaceborne SAR systems. The implementation of digital radar systems will moreover benefit from rapid developments in integrated microwaves and semi-conductor technologies [18]. For example, direct A/D conversion, which is already possible in L-band, simplifies the design of multi-channel receivers. In the long-term, one may even think about a multi-frequency SAR which employs the same digital receiver channels for different frequency bands. New semi-conductor technologies will furthermore pave the way to more advanced SAR systems with adaptive and hybrid imaging modes [13]. These sophisticated modes are well suited to resolve contradicting user requirements regarding resolution, coverage, and data acquisition continuity while taking into account limitations from both the available RF power and the downlink capacity.

\section{References}

[1] J. C. Curlander, R. N. McDonough, Synthetic Aperture Radar: Systems and Signal Processing. New York: Jon Wiley \& Sons, 1991.

[2] B. Jean, J. Rouse, "A multiple beam synthetic aperture radar design concept for geoscience applications," IEEE TGRS, vol. 21, 1983.

[3] A. Currie, M. A. Brown, "Wide-swath SAR," IEE Proceedings F Radar and Signal Processing, vol. 139, pp. 122-135, 1992.

[4] G. Callaghan, I. Longstaff, "Wide-swath space-borne SAR using a quad-element array,” IEE Proc. RSN, vol. 146, pp. 159-165, 1999.

[5] M. Suess, B. Grafmueller, R. Zahn, "A novel high resolution, wide swath SAR system,” Proc. IGARSS, Sydney, Australia, 2001.

[6] G. Krieger, N. Gebert, M. Younis, F. Bordoni, A. Patyuchenko, A. Moreira. „Advanced Concepts for Ultra-Wide-Swath SAR Imaging." , EUSAR 2008, pp. 31-34, Friedrichshafen, Germany, 2008.

[7] M. Younis, S. Huber, A. Patyuchenko, F. Bordoni, G. Krieger, „Performance Comparison of Reflector- and Planar-Antenna Based Digital Beam-Forming SAR”, IJAP, Article ID 614931, 2009.

[8] A. Freeman, G. Krieger, P. Rosen, M. Younis, W. Johnson, S. Huber, R. Jordan, A. Moreira. "SweepSAR: Beam-forming on Receive using a Reflector-Phased Array Feed Combination for Spaceborne SAR.” Proc. IEEE Radar Conf., Pasadena, USA, 2009.

[9] M. Spencer, S. Chan, L. Veilleux, K. Wheeler, "The Soil Moisture Active/Passive (SMAP) Mission Radar: a Novel Conically Scanning SAR”, Proc. IEEE Radar Conference, Pasadena, USA, 2009.

[10] A. Freeman et al., "Deformation, Ecosystem Structure, and Dynamics of Ice (DESDynI)", EUSAR, Frierichshafen, Germany, 2008.

[11] G. Krieger et al., "The Tandem-L Mission Proposal: Monitoring Earth's Dynamics with High Resolution SAR Interferometry," Proc. IEEE Radar Conf., Pasadena, USA, 2009.

[12] P. de Selding, "SkyTerra Might Launch 1 st of Two L-Band Satellites Early”, Space News, p. 14, March 2, 2009.

[13] G. Krieger, N. Gebert, A. Moreira, "Multidimensional Waveform Encoding: A New Digital Beamforming Technique for Synthetic Aperture Radar Remote Sensing," IEEE TGRS, vol. 46, pp. 31-46, 2008.

[14] S. Huber, M. Younis, A. Patyuchenko, G. Krieger, „A Novel Digital Beam-Forming Concept for Spaceborne Reflector SAR Systems", EURAD 2009, Rome, Italy.

[15] G. Krieger, I. Hajnsek, K. Papathanassiou, M. Younis, A. Moreira, "Single-Pass Synthetic Aperture Radar (SAR) Missions", Proc. IEEE - Special Issue on Satellite Remote Sensing Missions for Monitoring Water, Carbon and Global Climate Change, in revision.

[16] N. Gebert, G. Krieger, A. Moreira, "Multi-Channel Azimuth Processing in ScanSAR and TOPS Mode Operation", IEEE Trans. Geosc. Remote Sensing, in revision.

[17] N. Gebert, G. Krieger, "Ultra Wide Swath SAR Imaging with Continuous PRF Variation," EUSAR 2009.

[18] M. Ludwig, M. Suess, N. Le Gallou, "Technologies for advanced SAR systems”, EUSAR'06, Dresden, Germany, 2006. 